\title{
AVALIAÇÃO DA UTILIZAÇÃO DE INSTRUMENTAIS CIRÚRGICOS EM UM CENTRO CIRÚRGICO AMBULATORIAL
}

\author{
An evaluation of the use of surgical instruments in an Outpatient Surgery Center \\ Evaluación de la utilización de instrumentales quirúrgicos en un Centro Quirúrgico Ambulatorio \\ Adriana Breves dos Santos', Lucas Felix Calandrim', Joyce Marye Matsuoka Bidurim', \\ Raquel Aparecida Gonçalves', Cleuza Aparecida Vedovato², Ana Paula Boaventura ${ }^{3}$
}

RESUMO: Objetivo: Avaliar o número de instrumentais cirúrgicos utilizados e não utilizados durante cirurgias realizadas em um centro cirúrgico ambulatorial de um hospital universitário. Método: Trata-se de um estudo transversal, quantitativo e descritivo, realizado a partir do levantamento de dados com a observação da utilização ou não dos instrumentais cirúrgicos presentes nas caixas cirúrgicas. Resultados: Foram observadas 176 cirurgias, dentre as especialidades: oftalmologia 132 (75\%), otorrinolaringologia 16 (9,09\%), plástica 12 (6,81\%) e outras especialidades $16(9,09 \%)$. Verificou-se $49,10 \%$ de desperdício dos instrumentais, por estes não terem sido utilizados nas cirurgias ambulatoriais. Conclusão: Este estudo traz uma nova perspectiva sobre a atuação da enfermagem no Centro Cirúrgico Ambulatorial e sua responsabilidade perante o gerenciamento e controle de custos de uma instituição de saúde. Palavras-chave: Instrumentos cirúrgicos. Procedimentos cirúrgicos ambulatórios. Controle de custos.

ABSTRACT: Objective: To evaluate the number of surgical instruments that are used and go unused during surgeries performed at an outpatient surgery center of a university hospital. Method: This is a cross-sectional, quantitative, and descriptive study, carried out using a survey of data of observations related to the use or non-use of surgical instruments present in surgical boxes. Results: A total of 176 surgeries were observed among the specialties: ophthalmology 132 (75\%), otorhinolaryngology 16 (9.09\%), plastic surgery $12(6.81 \%)$, and other specialties $16(9.09 \%)$. It was confirmed that $49.10 \%$ of the instruments were wasted, as they were not used in the outpatient surgeries. Conclusion: This study brings a new perspective about the role of nursing in Outpatient Surgery Centers and responsibility of nurses with regard to the management and control of costs at health institutions.

Keywords: Surgical Instruments, Outpatient Surgical Procedures, Cost control.

RESUMEN: Objetivo: Evaluar el número de instrumentales quirúrgicos utilizados y no utilizados durante cirugías realizadas en un centro quirúrgico ambulatorio de un hospital universitario. Método: Se trata de un estudio transversal, cuantitativo y descriptivo, realizado a partir del levantamiento de datos con la observación de la utilización o no de los instrumentales quirúrgicos presentes en las cajas quirúrgicas. Resultados: Fueron observadas 176 cirugías, entre las especialidades: oftalmología 132 (75\%), otorrinolaringología 16 (9,09\%), plástica 12 (6,81\%) y otras especialidades 16 (9,09\%). Se verificó un $49,10 \%$ de desperdicio de los instrumentales, por estos no haber sido utilizados en las cirugías ambulatorias. Conclusión: Este estudio trae una nueva perspectiva sobre la actuación de la enfermería en el Centro Quirúrgico Ambulatorio y su responsabilidad ante la gestión y control de costos de una institución de salud.

Palabras clave: Instrumentos Quirúrgicos, Procedimientos Quirúrgicos Ambulatorios, Control de Costos.

'Alunos do curso de Graduação em Enfermagem da Faculdade de Enfermagem da Universidade Estadual de Campinas (UNICAMP). E-mails: adrianabreves@gmail.com, lucas.calandrim@gmail.com, jbidurim@gmail.com, goncalvesraquel10@gmail.com

¿Enfermeira mestre na Faculdade de Enfermagem da UNICAMP. E-mail: vedovato@unicamp.br

${ }^{3}$ Professora doutora na Faculdade de Enfermagem da UNICAMP. E-mail: apboa@unicamp.br

Avenida José Puccinelli, 10 (Rua 6 casa 92) - Cascata - CEP: 13146-000 - Paulínia (SP), Brasil.

Recebido: 29 nov. 2016 - Aprovado: 8 mar. 2017

DOI: $10.5327 / 21414-4425201700020004$ 


\section{INTRODUÇÃO}

A cirurgia ambulatorial, no contexto mundial, está ligada à trajetória da história da enfermagem e tornou-se uma realidade com os progressivos avanços das técnicas cirúrgicas minimamente invasivas e das drogas anestésicas que possibilitaram uma recuperação rápida do paciente, com mínimas complicações ${ }^{1,2}$.

O objetivo principal de um centro cirúrgico ambulatorial é a segurança para a realização das cirurgias, com baixo custo e que permita que o paciente fique no hospital por um curto período de tempo ${ }^{2}$.

O Centro Cirúrgico Ambulatorial (CCA) é muito parecido com o Centro Cirúrgico (CC) tradicional, diferindo pelo fato de a alta do paciente ocorrer no mesmo dia do procedimento, repercutindo em uma série de vantagens como a redução da ansiedade, menor risco de se contrair infecção hospitalar, diminuição do tempo de recuperação, além da economia para o hospital ${ }^{1-2}$.

As cirurgias que podem ser realizadas no CCA são de pequena complexidade, mediante técnicas anestésicas local, locorregional, de bloqueio (raqui e/ ou peridural) ou, ainda, a geral (inalatória e/ou endovenosa). Os pacientes devem ter ausência de comprometimento sistêmico - por outras doenças ou pela cirurgia - , e serão submetidos a procedimentos cirúrgicos que não precisem de cuidados específicos no pós-operatório e obrigatoriamente devem ter acompanhante para alta ${ }^{1-3}$.

Um fator de importante atuação para garantir a assistência de enfermagem transoperatória em um CC é a interface necessária com o Centro de Materiais e Esterilização (CME), que provê todos os materiais e instrumentais esterilizados utilizados em cada procedimento cirúrgico.

O controle da utilização dos instrumentais cirúrgicos dentro da sala operatória constitui primordial função da equipe de enfermagem, a fim de garantir a segurança do paciente e da equipe médica para adequada realização da técnica cirúrgica proposta. O profissional de enfermagem recebe o material do CME, faz a conferência inicial dos instrumentais presentes em sala, e, ao final da cirurgia, confere a integridade dos instrumentais e faz a devolução de todos os instrumentais para novo processamento no $\mathrm{CME}^{4-6}$.

Instrumentais cirúrgicos e outros materiais, utilizados no ambiente hospitalar, são tidos como recursos materiais e representam $75 \%$ do capital das organizações. Portanto, a forma de administrá-lo reflete diretamente nos custos da empresa $^{7,8}$.
A quantidade de recursos materiais, especificamente de instrumentais cirúrgicos, deve ser contabilizada pelo enfermeiro, proporcionando o desenvolvimento dos serviços sem imprevistos, evitando desperdícios e custo elevado ${ }^{7,8}$.

Este estudo envolve importante etapa da assistência de transoperatória de enfermagem, em que o paciente deve ser rigorosamente avaliado e acompanhado dentro de sala operatória e o controle de todos os processos e procedimentos que serão realizados em sala ao longo de toda a cirurgia, sobretudo o controle e avaliação dos instrumentais e materiais que serão utilizados nesta fase, são atribuições de responsabilidade do enfermeiro do $\mathrm{CC}^{1}$.

\section{OBJETIVO}

Avaliar o número de instrumentais cirúrgicos utilizados e não utilizados durante cirurgias realizadas em um CCA de um hospital universitário.

\section{MÉTODOS}

Foi desenvolvido um estudo quantitativo e descritivo, realizado a partir do levantamento de dados com a observação sistematizada da utilização ou não dos instrumentais cirúrgicos presentes nas caixas cirúrgicas.

O local de estudo foi o CCA de um hospital universitário, localizado no interior do Estado de São Paulo, com 411 leitos, de nível terciário e quaternário, onde todos os atendimentos são realizados e pagos integralmente pelo Sistema Único de Saúde (SUS). O CCA realiza em média 600 cirurgias por mês, distribuídas em 8 salas operatórias destinadas a algumas especialidades como oftalmologia, otorrinolaringologia, dermatologia, cirurgia plástica, cabeça e pescoço, neurologia e urologia.

Os dados foram coletados após análise e aprovação do Comitê de Ética da Universidade Estadual de Campinas sob o parecer consubstanciado de número 1.384.178 de 06/01 / 2016. A coleta de dados foi autorizada pela diretoria de enfermagem do CCA e pelos enfermeiros responsáveis pelo local após explicação dos objetivos da pesquisa, com leitura e assinatura do Termo de Consentimento Livre e Esclarecido, garantindo dessa forma os preceitos éticos e legais envolvidos em pesquisa com seres humanos, respeitando assim os aspectos ético-legais da Resolução n ${ }^{\circ}$ 466/2012, do Comitê Nacional de Pesquisas com Seres Humanos. 
O instrumento de coleta de dados consistiu nas listas de instrumentais cirúrgicos contidos nas caixas cirúrgicas das cirurgias realizadas no CCA. Algumas especialidades, como a oftalmologia, utilizam caixas cirúrgicas dos próprios cirurgiões, as quais não possuem listagem. Para coleta de dados referentes às cirurgias que utilizavam caixas cirúrgicas sem listagem, observou-se a quantidade de instrumentais totais na caixa cirúrgica e a quantidade de instrumentais utilizados durante a cirurgia. Cirurgias do segundo trimestre de 2016 foram acompanhadas do início ao fim para a avaliação fidedigna dos instrumentais cirúrgicos utilizados e não utilizados.

Os dados foram coletados pela pesquisadora, armazenados em uma planilha, tabulados com o auxílio do programa Microsoft Excel ${ }^{\circledR}$ e analisados sob orientação estatística.

O cálculo amostral foi realizado considerando o objetivo de estimar a proporção de instrumentais cirúrgicos não utilizados nas cirurgias do CCA, em um período de três meses. O cálculo amostral considerou uma proporção $p$ igual a 0,50, cujo valor representa a variabilidade máxima da distribuição binomial, gerando assim uma estimativa com o maior tamanho amostral possível.

A população $(\mathrm{N})$ considerada para o cálculo do tamanho amostral era composta de 1.629 cirurgias ambulatoriais, realizadas no período de fevereiro a abril de 2015. Além disso, foi assumido um erro amostral de $5 \%$ e um nível de significância de 5\%. Com isso, o tamanho amostral calculado foi de 176 cirurgias. Essa amostra foi dividida, proporcionalmente, de acordo com o número de cirurgias realizadas e com as especialidades das cirurgias.

\section{RESULTADOS}

O CCA deste hospital realizou 7.196 cirurgias ambulatoriais no ano de 2016, e 1.659 cirurgias no segundo trimestre desse mesmo ano, período em que fora coletados os dados deste estudo.
Durante o período de coleta de dados, e conforme o cálculo amostral, foram observadas 176 cirurgias, que correspondem a $10,6 \%$ do total das cirurgias realizadas no segundo trimestre, dentre as especialidades: oftalmologia 132 (75\%), otorrinolaringologia $16(9,09 \%)$, plástica $12(6,81 \%)$ e outras especialidades $16(9,09 \%)$, como cabeça e pescoço (6), dermatologia (8), neuroclínica (1) e urologia (1).

A quantidade de caixas cirúrgicas abertas durante as cirurgias variou de 1 a 4 , sendo utilizada apenas uma caixa em 145 cirurgias $(82,38 \%)$.

Em 176 cirurgias coletadas no CCA, verificou-se que 132 (75\%) são cirurgias oftalmológicas e foi apresentada uma média de 18,17 instrumentais em cada uma das cirurgias. Já para a realização das cirurgias da otorrinolaringologia 16 (9,09\%), verificou-se em média 40,19 instrumentais na sala operatória.

Verifica-se ainda (Tabela 1) que, dentre essas cirurgias de pequeno porte realizadas ambulatorialmente, há procedimentos com apenas um instrumental e outros com até 86 instrumentais.

Verificou-se na Tabela 2 que as cirurgias otorrinolaringológicas apresentam uma média de 27,06 instrumentais não utilizados, enquanto na especialidade de oftalmologia a média de instrumentais não utilizados é de 9,55.

Dentre as 176 cirurgias analisadas verificou-se que, em média, 11,67 instrumentais não são utilizados em cirurgias ambulatoriais.

Verificou-se também (Tabela 3), neste estudo, que houve uma média geral de 49,10\% instrumentais não utilizados em cirurgias ambulatoriais.

\section{DISCUSSÃO}

Um CCA atua diariamente realizando procedimentos cirúrgicos de baixa e média complexidade de diversas especialidades. A assistência de enfermagem perioperatória inclui a avaliação da segurança do paciente, dos custos e dos resultados dos

Tabela 1. Distribuição de instrumentais nas caixas cirúrgicas utilizadas no Centro Cirúrgico Ambulatorial. Campinas, 2016. (n=176).

\begin{tabular}{|l|c|c|c|c|c|c|}
\hline Variável & Total de cirurgias por especialidade & $\mathbf{n}$ & Média & Desvio-padrão & Mínimo & Máximo \\
\hline & Oftalmologia & 132 & 18,17 & 8,46 & 1 \\
\hline \multirow{2}{*}{ Total de instrumentais } & Otorrinolaringologia & 16 & 40,19 & 21,98 & 13 \\
\hline & Cirurgia plástica & 12 & 23,83 & 10,22 & 8 \\
\hline & Outras & 16 & 24,44 & 5,82 & 12 \\
\hline
\end{tabular}


cuidados ministrados. Além disso, é de responsabilidade do enfermeiro gerenciar recursos humanos e materiais devido à sua formação, que lhe garante competências éticas e técnicas nesse aspecto ${ }^{9}$.

Nas 176 cirurgias verificou-se que, em média, haviam 21,13 instrumentais nas salas operatórias. Houve cirurgias em que a quantidade de instrumentais chegou a 86. Considerando que os instrumentais não utilizados foram em média 11,67 instrumentais no geral, chegando a 27,06 na especialidade de otorrinolaringologia, questiona-se a quantidade elevada dos mesmos presentes nas salas cirúrgicas.

A partir do momento que a caixa cirúrgica é aberta dentro das salas cirúrgicas - consequentemente expondo os instrumentais - , é necessário que estes sejam encaminhados ao CME, onde sofrerão processamento que os restituirá esterilizados ${ }^{6}$. Tal processo, realizado sem necessidade, pode levar ao desgaste do material e a custos dispendiosos para a instituição de saúde.

O presente estudo demonstrou que, em média, 49\% dos instrumentais cirúrgicos não são utilizados durante as cirurgias no CCA. Tal número é considerado elevado devido a estes instrumentais terem que passar novamente por processo de esterilização, acarretando maiores custos à instituição, há gastos com insumos, mão de obra para realizar a limpeza dos instrumentais, sua embalagem e estocagem; há também gastos com o consumo de água, energia elétrica e manutenção da esterilizadora ${ }^{10}$.

Por representarem um grande investimento para a instituição, os instrumentais cirúrgicos devem ser usados adequadamente para que mantenham sua qualidade e tenham sua vida prolongada. Cabe ressaltar que as organizações de saúde, devido aos recursos limitados e aos custos assistenciais elevados, precisam encontrar alternativas para reduzir os gastos e aumentar a produtividade, de modo que haja redução do desperdício ${ }^{10-12}$.

Os motivos pelos quais estes instrumentais não são utilizados são: o número em excesso de instrumentais na composição das caixas cirúrgicas previamente estabelecidas pela equipe cirúrgica, que realmente não serão necessários no procedimento cirúrgico proposto; a simplicidade dos procedimentos ambulatoriais e a evolução das técnicas cirúrgicas fazendo com que muitos instrumentais deixem de ser utilizados; bem como a preferência por instrumentais específicos que não compõem a caixa da cirurgia proposta, sendo solicitada caixa de outra especialidade ou particular da equipe cirúrgica para a utilização de instrumentais extremamente

Tabela 2. Distribuição dos instrumentais utilizados e não utilizados no Centro Cirúrgico Ambulatorial. Campinas, 2016. (n=176).

\begin{tabular}{|c|c|c|c|c|c|c|}
\hline Variável & Especialidade & $\mathbf{n}$ & Média & Desvio-padrão & Mínimo & Máximo \\
\hline \multirow{5}{*}{ Instrumentais utilizados } & Oftalmologia & 132 & 8,61 & 3,74 & 1 & 21 \\
\hline & Otorrinolaringologia & 16 & 13,13 & 8,2 & 1 & 32 \\
\hline & Cirurgia plástica & 12 & 9,5 & 5,47 & 3 & 21 \\
\hline & Outras & 16 & 12,69 & 4,56 & 5 & 19 \\
\hline & Total & 176 & 9,45 & 4,75 & 1 & 32 \\
\hline \multirow{5}{*}{ Instrumentais não utilizados } & Oftalmologia & 132 & 9,55 & 7,51 & 0 & 47 \\
\hline & Otorrinolaringologia & 16 & 27,06 & 16,62 & 5 & 64 \\
\hline & Cirurgia plástica & 12 & 14,33 & 7,94 & 4 & 23 \\
\hline & Outras & 16 & 11,75 & 6,28 & 2 & 27 \\
\hline & Total & 176 & 11,67 & 9,94 & 0 & 64 \\
\hline
\end{tabular}

Tabela 3. Percentual dos instrumentais não utilizados nas cirurgias realizadas no Centro Cirúrgico Ambulatorial. Campinas, 2016 ( $\mathrm{n}=176$ ).

\begin{tabular}{|l|c|c|c|c|c|c|}
\hline Variável & Especialidade & $\mathbf{n}$ & Média & Desvio-padrão & Mínimo & Máximo \\
\hline & Oftalmologia & 132 & 46,4 & 23,28 & 0 & 86,67 \\
\hline \multirow{2}{*}{ Percentual de desperdício } & Otorrinolaringologia & 16 & 66,49 & 14,9 & 38,46 & 93,75 \\
\hline & Cirurgia plástica & 12 & 58,35 & 16,99 & 33,33 & 87,5 \\
\hline & Outras & 16 & 46,97 & 19,39 & 10 & 78,26 \\
\hline & Total & 176 & 49,1 & 22,68 & 0 & 93,75 \\
\hline
\end{tabular}


específicos. Assim, evidencia-se que há desaproveitamento dos instrumentais, o que acarreta em custos desnecessários para a instituição, contudo tais custos podem ser previstos e corrigidos ${ }^{13,14}$.

O enfermeiro é o responsável por realizar o controle do material, assim como fazer a comunicação entre as unidades para haver um menor custo na hora do processamento, sendo que muitas vezes a prática de substituição do instrumental é demorada e burocrática, repercutindo em mais custos ${ }^{14,15}$.

O CME é responsável por garantir a reutilização segura dos instrumentais, realizando o processamento dos mesmos, e verificando também o desempenho dos instrumentais por meio de testes específicos, a fim de garantir maior segurança ao paciente e aos profissionais ${ }^{16,17}$.

Cabe ao enfermeiro pesquisar novas alternativas e soluções para o problema da não utilização de instrumentais cirúrgicos, tendo como objetivo principal a redução de custos desnecessários à instituição. Para tal, é primordial a identificação dos desperdícios e a necessidade de implementar um trabalho de conscientização e mudanças comportamentais no trabalho da equipe que atua no centro cirúrgico ambulatorial, utilizando para isso a educação permanente em serviço com foco nos processos de trabalho, com a finalidade de aperfeiçoar e desenvolver os profissionais visando a redução dos desperdícios e estratégias para minimizá-las ${ }^{16,17}$.

Com esse intuito propõe-se a revisão de processos de trabalho que integrem CCA e CME, bem como que envolvam tanto a equipe de enfermagem como a equipe médica, propondo reformulação na composição das caixas cirúrgicas específicas de modo que possam acompanhar a evolução das técnicas cirúrgicas modernas ${ }^{16,17}$.

\section{CONCLUSÃO}

A média geral de instrumentais não utilizados nas cirurgias ambulatoriais nesse centro cirúrgico foi de $49 \%$, com predomínio de cirurgias da especialidade de oftalmologia, sendo utilizada, em sua maioria, uma caixa cirúrgica por procedimento com média de 21,13 instrumentais por caixa. Verificou-se que o enfermeiro é o profissional que atua na gestão dos recursos materiais no CCA, impactando no controle de custos da instituição e na busca de melhores estratégias que visem melhorias nos processos de trabalho.

Destaca-se a importância da integração entre CCA e CME, o que possibilitaria a revisão dos instrumentais contidos nas caixas cirúrgicas, bem como a elaboração de kits de instrumentais específicos para determinados procedimentos ou instrumentais específicos avulsos.

Apontam-se como limitação deste estudo poucas publicações sobre esse tema tanto na literatura nacional quanto na estrangeira, o que dificulta realizar a comparação de resultados. Desse modo, os resultados encontrados não se aplicam a todas as instituições. Mesmo assim, o estudo atingiu seu objetivo e se destacou por identificar o percentual de desperdício de instrumentais cirúrgicos, contribuindo para o planejamento de revisão dos processos de trabalho técnico e administrativos, visando um maior aproveitamento e utilização dos instrumentais cirúrgicos, bem como a redução de custos para instituições de saúde. Envolve também uma nova perspectiva para futuros estudos sobre a atuação da enfermagem no CCA e sua responsabilidade perante o gerenciamento e controle de recursos materiais nas instituições de saúde.

\section{REFERÊNCIAS}

1. Sociedade Brasileira de Enfermagem de Centro Cirúrgico. Recuperação Anestésica e Central de Materiais e Esterilização. Práticas Recomendadas. SOBECC. 6. ed. São Paulo: SOBECC; 2013.

2. Santos JS, Sankarankutty AK, Salgado Jr. W, Kemp R, Leonel EP, Castro e Silva Jr. O. Cirurgia ambulatorial: do conceito à organização de serviços e seus resultados. Med [Internet]. 2008 [citado em 2016 fev. 14];41(3):274-86. Disponível em: http://revista.fmrp.usp.br/2008/ VOL41N3/SIMP_4Cirurgia_ambulatorial pdf
3. Figueiredo NMA, Leite JL, Machado WCA. Centro cirúrgico: atuação, intervenção e cuidados de enfermagem. 2 ed. Coletânea de Enfermagem Digital: trinta e duas grandes obras da Yendis. São Caetano do Sul: Editora Yendis; 2009. 1 CD-ROM.

4. Parra OM, Saad WA. Instrumentação Cirúrgica. 3. ed. São Paulo: Atheneu; 1988.

5. Klenger FJ. Administração hospitalar. Goiânia: AB; 2002. 
6. Moriya T, Vicente YMVA, Tazima MFGS. Instrumental cirúrgico. Med [Internet]. 2011 [citado em 2016 fev. 14];44(1):18-32. Disponível em: http://revista. fmrp.usp.br/2011/vol44n1/Simp2_Instrumental\%20cir\%FArgico.pdf

7. Freitas LR, Tipple AFV, Pires FV, Melo DS, Spagnoli JLU. (Des)cuidado com produtos para saúde processados no transporte e armazenamento em unidades de internação. Texto Contexto - Enferm [Internet]. 2015 [citado em 2016 fev. 16];24(1):253-262. Disponível em: http://www.scielo. br/scielo.php?script=sci_arttext\&pid=S0104-07072015000100253\&lng $=\mathrm{pt} \& \mathrm{nrm}=\mathrm{iso} \& \mathrm{tlng}=\mathrm{pt}$

8. Jericó MC, Castilho V. Gerenciamento de custos: aplicação do método de custeio baseado em atividades em centro de material esterilizado. Rev Esc Enferm USP [Internet]. 2010 [citado em 2016 fev. 16];44(3); 745-52. Disponivel em: http://www.scielo.br/pdf/reeusp/v44n3/28.pdf

9. Rothrock JC. Conceitos básicos de enfermagem perioperatória. In: Rothrock JC. Cuidados de enfermagem ao paciente cirúrgico. Rio de Janeiro: Elsevier; 2007.

10. Castro LC, Castilho V. 0 custo de desperdício de materiais de consumo em um centro cirúrgico. Rev Latino-Am Enferm [Internet]. 2013 [citado em 2016 fev. 16];21(6):1228-34. Disponível em: http://www.scielo.br/ scielo.php?script=sci_arttext\&pid=S0104-11692013000601228\&lng $=e n \& n r m=i s o \& t \operatorname{lng}=p t$

11. Paula JRA, Silva RCR, Vedovato CA, Boaventura AP. Instrumentais nas caixas cirúrgicas: avaliação de custo. Rev SOBECC [Internet]. 2015 [citado em 2016 fev. 23];20(2):73-80. Disponível em: http:// www.sobecc.org.br/arquivos/artigos/2015/pdfs/v20n2/73-80.pdf

12. Grossi MG, Bittar E. A substituição de materiais de consumo na dinâmica de trabalho do enfermeiro em um hospital cardiológico.
Rev Adm Hosp Inov Saúde [Internet]. 2011 [citado em 2016 abr. 24];8(8). Disponivel em: http://revistas.face.ufmg.br/index.php/rahis/ article/view/1560/987

13. Costa EA, Dórea EO, Alves MA, Nery F, Schettini H, Belmonte M, et al. Reprocessamento de produtos para saúde: análise da qualidade sanitária em hospitais públicos. Rev SOBECC [Internet]. 2015 [citado em 2016 abr. 23];20(1):17-23. Disponível em: http://www.sobecc. org.br/arquivos/artigos/2015/pdfs/v20n1/v20n1_17-23.pdf

14. Ascari RA, Vidori J, Moretti CA, Perin EMF, Silva OM, Buss E. O processo de esterilização de materiais em serviços de saúde: uma revisão integrativa. Braz J Surg Clin Res [Internet]. 2013 [citado em 2016 out. 16];4(2):33-38. Disponível em: http://www.mastereditora.com. br/periodico/20130831_181149.pdf

15. Ouriques CM, Machado ME. Enfermagem no processo de esterilização de materiais. Texto Contexto - Enferm [Internet]. 2013 [citado em 2016 out. 16];22(3):695-703. Disponível em: http://www.scielo.br/ scielo.php?script=sci_arttext\&pid=S0104-07072013000300016\&lng $=\mathrm{pt \& nrm}=\mathrm{iso}$

16. Lima RS, Lourenço EB, Rosado SR, FavaSMCL, Sanches RS, Dázio EMR. Representação da prática gerencial do enfermeiro naunidade de internação: perspectiva da equipe de enfermagem. Rev Gaúcha Enferm [Internet]. 2016 [citado em 2016 out. 16];37(1). Disponível em: http://www.scielo. br/scielo.php?script=sci_arttext\&pid=S1983-14472016000100406\&lng $=$ en\&nrm=iso

17. Silva MJN, Ribeiro AL. Gestão em centro cirúrgico: identificação de desperdícios. Rev SOBECC [Internet]. 2016 [citado em 2016 out. 16];21 (2):82-9. Disponível em: https://revista.sobecc.org.br/sobecc/ article/view/120 